\title{
ScannerS: parameter scans in extended scalar sectors
}

\author{
Margarete Mühlleitner ${ }^{1, a}$, Marco O. P. Sampaio ${ }^{2, b}$, Rui Santos ${ }^{3,4, c}$, Jonas Wittbrodt ${ }^{5, d}$ (i) \\ ${ }^{1}$ Institute for Theoretical Physics, Karlsruhe Institute of Technology, 76128 Karlsruhe, Germany \\ ${ }^{2}$ Feedzai-Porto, Rua de Santos Pousada 228 Piso 4, 4000-278 Porto, Portugal \\ ${ }^{3}$ Centro de Física Teórica e Computacional, Faculdade de Ciêncas, Universidade de Lisboa, campo Grande, Edifício C8, 1749-016 Lisbon, \\ Portugal \\ ${ }^{4}$ ISEL-Instituto Superior de Engenharia de Lisboa, Instituto Politécnico de Lisboa, 1959-007 Lisbon, Portugal \\ ${ }^{5}$ Department of Astronomy and Theoretical Physics, Lund University, Sölvegatan 14A, 22362 Lund, Sweden
}

Received: 5 November 2021 / Accepted: 14 February 2022 / Published online: 5 March 2022

(C) The Author(s) 2022

\begin{abstract}
We present the public code ScannerS-2 that performs parameter scans and checks parameter points in theories beyond the Standard Model (BSM) with extended scalar sectors. ScannerS incorporates theoretical and experimental constraints from many different sources in order to judge whether a parameter point is allowed or excluded at approximately $95 \% \mathrm{CL}$. The BSM models implemented in ScannerS include many popular BSM models such as singlet extensions, different versions of the Two-Higgs-Doublet Model, or the different phases of the Next-to Two-HiggsDoublet Model. The ScannerS framework allows straightforward extensions by additional constraints and BSM models.
\end{abstract}

\section{Contents}

1 Introduction . . . . . . . . . . . . . 1

2 Constraints ................ 2

2.1 Theoretical constraints . . . . . . . . . 3

2.1.1 Perturbative unitarity . . . . . . . . . 3

2.1.2 Boundedness from below . . . . . . . . . 3

2.1.3 Vacuum stability . . . . . . . . . 3

2.2 Electroweak precision constraints . . . . . 3

2.3 Flavour constraints . . . . . . . . . . . 4

2.4 Higgs searches and Higgs measurements . . . . 4

2.5 Electric dipole moments . . . . . . . . . . 4

2.6 DM constraints . . . . . . . . . . . . . . . 4

2.7 A first order EW phase transition . . . . . . . 5

3 BSM Models in ScannerS . . . . . . . . . . 5

a e-mail: milada.muehlleitner@kit.edu

be-mail: marco.sampaio@feedzai.com

c e-mail: rasantos@fc.ul.pt

d e-mail: jonas.wittbrodt@ thep.lu.se (corresponding author)
3.0.1 Input parameters and mass-ordering . . . 5

3.1 The complex-singlet-extension of the SM . . . 6 3.1.1 The broken-phase CxSM - CxSMBroken 7

3.1.2 The dark-phase CxSM - CxSMDark . . 7

3.2 The two-real-singlet-extension of the SM . . . 7 3.2.1 The broken-phase TRSM - TRSMBroken 7

3.3 The two-Higgs-doublet model . . . . . . . 7

3.3.1 The R2HDM - R2HDM . . . . . . . . 8

3.3.2 The C2HDM-C2HDM . . . . . . . 8

3.4 The next-to $2 \mathrm{HDM}$. . . . . . . . . . . . . . . 9

3.4.1 The broken-phase N2HDM - N2HDMBroken . 10

3.4.2 The dark-singlet-phase N2HDM - N2HDMDarkS 10

3.4.3 The dark-doublet-phase N2HDM - N2HDMDarkD 10

3.4.4 The fully-dark-phase N2HDM - N2HDMDarkSD 11

3.5 Minimal CP-violating dark matter-CPVDM . . 11

4 User operating instructions . . . . . . . . . . . . . 12

4.1 Extending Scanners . . . . . . . . . . . . 12

5 Summary . . . . . . . . . . . . . . . . . . 13

A The anyHdecay library . . . . . . . . . . . . . 13

B Perturbative unitarity bounds . . . . . . . . . . . . . . . . . 14

References . . . . . . . . . . . . . . . . 15

\section{Introduction}

In the exploration of extensions of the Standard Model (SM) of particle physics it is mandatory to work with allowed parameter points - points within the parameter space of the model that do not disagree with current observations. Such samples of allowed parameter points can be used to showcase the possible phenomenology in a beyond the SM (BSM) theory or to illustrate the impact of novel calculations for a physically relevant scenario. The code ScannerS [1] can be used to generate and validate samples of such allowed parameter points in many BSM models with extended scalar sectors. 
All of the BSM models implemented in ScannerS are pure electroweak (EW) scalar extensions of the SM. In particular, ScannerS does not target supersymmetric models or any other BSM models that introduce new colored scalars. The models implemented in ScannerS range from simple singlet extensions of the SM, through the commonly used variants of the two-Higgs-doublet model (2HDM) to extensions by both additional doublets and scalar singlets. We have found these models to be extremely useful as benchmark models for both collider physics and particle cosmology, but also as well understood reference models to study the impact of novel theoretical calculations. We specifically invite users to implement their own models of interest within the ScannerS framework and make those implementations available to the community.

ScannerS performs parameter scans within the parameter space of the model. A parameter scan is a greatly simplified model fit that forgoes making any statements about the model as a whole in favor of a simpler, point-by-point approach. In model fits - such as those performed with the Fittino [2], ZFitter [3], GFitter [4], GAMBIT [5], or HEPFIT [6] public codes - an overall combined $\chi^{2}$ or likelihood is constructed from all available measurements. By sampling this distribution and using the resulting best fit point of the model, statements about the favoured regions of parameter space and the compatibility between model predictions and data can be made. ${ }^{1}$ However, there are many cases where no global statements about the model are required. In these cases performing a full model fit is often excessive and a simpler approach is sufficient.

The parameter scan approach employed in ScannerS does not construct a global likelihood distribution but instead uses a set of individual constraints. This means, that ScannerS tests the model predictions for the (randomly generated) input parameter points against all implemented constraints and treats each parameter point that passes all constraints as allowed. This point-by-point approach means that no overall best-fit-point is found, nor is the resulting sample of allowed parameter points a faithful representation of the global likelihood distribution. These limitations mean that the results of a parameter scan should never be used to make global statements about the model - such as "the model fits the data well" or "the model cannot explain this observation". Furthermore, no conclusions should be drawn based on the density of the allowed parameter points in the parameter space of the model. This would require the points to follow a statistically meaningful distribution while, in a parameter scan, their distribution entirely depends on the sampling.

On the other hand, the results of a parameter scan are perfectly suited for phenomenological benchmark scenar-

\footnotetext{
1 The exact statements possible depend on the chosen statistical interpretation - frequentist, bayesian, or some mixture of the two.
}

ios, illustrating interesting signatures, checking the effects of precision calculations, and many other applications that only rely on the existence of allowed parameter points in some region of parameter space. Since the naive combination of the individual constraints leads to an overestimated combined constraint, the region covered by the allowed parameter points is a conservative estimate for the favoured region that would be found in a model fit. ${ }^{2}$ The most important advantage of parameter scans is simplicity. Since no best fit point is found or needed ${ }^{3}$ it is perfectly fine to run small parameter scans that only yield a few allowed parameter points or to focus on some special regions of the parameter space that may not necessarily contain the best fit point of the model. Additionally, the non-reliance on the best fit point means that an existing sample of parameter points can simply be re-checked with ScannerS if some constraints are updated and no new scan is required.

This manual discusses the physics of ScannerS and accompanies the release of ScannerS-2. It is complemented by the technical documentation available online at https:// jonaswittbrodt.gitlab.io/ScannerS.

ScannerS-2 is a new code inspired by the old ScannerS-1 [1]. Compared to the old code, we implemented substantial technical upgrades and many physics improvements. In this manual we first discuss the constraints included in ScannerS in Sect. 2. In Sect. 3 we then give short overviews of the implemented BSM models to establish the conventions used in the code. Section 4 contains instructions on how to build and use ScannerS and an explanation on how additional models and constraints can be implemented. We summarize in Sect. 5. In Appendix A we describe the anyHdecay interface library and in Appendix B we discuss the Mathematica package for finding perturbative unitarity constraints that is distributed with ScannerS.

\section{Constraints}

Experimental constraints in ScannerS are implemented at a confidence level (CL) of $95 \%$ or - almost equivalently $-2 \sigma$ while theoretical constraints are simple exclusions without an associated statistical interpretation. As such, the allowed parameter points returned by ScannerS are valid from a theoretical point of view and not excluded by observations

\footnotetext{
$\overline{2}$ This only holds as long as correlations between different constraints are small. In ScannerS, most constraints stem from distinct sources and can be assumed uncorrelated to a good approximation.

${ }^{3}$ In the constraints that are reconstructed as a $\chi^{2}$ value - notably the oblique parameters and the Higgs measurements - ScannerS applies the constraint with the SM as alternative hypothesis.
} 
at approximately $95 \%$ CL. ${ }^{4}$ Constraints in ScannerS have one of three different severities governing how their result is handled by the code. The strongest and default severity is apply, meaning that only parameter points fulfilling the constraints will be kept as allowed points. If the weakest severity, skip, is set for a constraint, no calculations associated with it will be performed and parameter points will not be tested against it. For a constraint with the intermediate severity, ignore, all associated calculations will be executed and the results saved to the output. However, parameter points will be treated as allowed whether they fulfill the constraint or not, while an additional output value will be added to indicate if the specific constraint was fulfilled. Practical information on setting severities is given in Sect. 4.

Some of the following constraints are not applicable to all of the models implemented in ScannerS. Detailed technical information regarding the implementation of constraints in ScannerS can be found in the online documentation for the ScannerS: : Constraints namespace. The online documentation also lists all output quantities associated to the constraints.

\subsection{Theoretical constraints}

Theoretical constraints are self-consistency requirements on a parameter point of the model. As such, they do not have a statistical interpretation - they are either fulfilled or the parameter point is invalid and excluded.

\subsubsection{Perturbative unitarity}

If a theory violates unitarity it is strongly coupled and cannot be treated in the perturbative approach employed in ScannerS. Unitarity constraints, are obtained by requiring the eigenvalues $\mathcal{M}_{2 \rightarrow 2}^{i}$ of the $2 \rightarrow 2$ scattering matrix $\mathcal{M}_{2 \rightarrow 2}$ to fulfill

$$
\left|\mathcal{M}_{2 \rightarrow 2}^{i}\right| \leq 8 \pi \text {. }
$$

The tree-level scattering matrix can be easily constructed in most BSM models with extended Higgs sectors in the high energy limit $[7,8]$. It often has a block-diagonal form that can - at least partially - be diagonalized analytically. Whenever possible the corresponding closed form solutions for the eigenvalues are implemented in ScannerS.

Tree-level perturbative unitarity provides only a first approximation to the true unitarity constraint on the model parameters. It may be impacted by loop-corrections [9] or finite-energy effects [10-12].

\footnotetext{
4 The overall constraint is in fact slightly stronger than 95\% CL due to the naive combination of constraints. Additionally, all correlations between constraints are neglected.
}

\subsubsection{Boundedness from below}

Boundedness of the scalar potential from below is a prerequisite to the existence of a stable vacuum. ScannerS implements analytical conditions that ensure boundedness from below at tree level, whenever possible. In general, such conditions can be very challenging to obtain (see e.g. Ref. [13] for a recent overview of different methods and their applications). However, closed form solutions have been found for many BSM models - including all of the models implemented in ScannerS, see Sect. 3.

\subsubsection{Vacuum stability}

Even if the scalar potential is bounded from below, the EW vacuum is not necessarily the global minimum (absolute stability). In this case the lifetime of the EW vacuum with respect to vacuum decay must be long-lived compared to the age of the universe, otherwise the vacuum is unstable.

In some models or phases of models, absolute stability of the tree-level EW vacuum has been proven, or analytic conditions ensuring stability have been found (see also Sect. 3). For the remaining cases, ScannerS offers a link to the EVADE library [14-16] to test the stability of the EW vacuum numerically at tree-level. Since the theoretical uncertainties involved in this computation are large and hard to estimate we are very conservative in the application of this constraint and only consider points unstable and excluded if the survival probability of the EW vacuum is less than $5 \sigma \approx 3 \times 10^{-7}$. See the discussion in Ref. [14] for more details.

\subsection{Electroweak precision constraints}

Precision measurements of EW observables are sensitive to BSM loop effects present in many extended scalar sectors. As long as the BSM effects are fully captured through selfenergy corrections to the gauge-boson propagators - which holds in all models discussed here - and the new physics scale is not too small, these effects can be parametrized through the oblique parameters $S, T$, and $U$ [17]. ScannerS does not compute any EW precision observables and instead uses a fit result-currently from Ref. [18] - for the values of the oblique parameters as constraint. This result includes a covariance matrix to account for correlations among $S, T$, and $U$. In order to calculate model predictions for the oblique parameters ScannerS implements the results of Refs. [19,20], which are valid for models with any number of scalar $\mathrm{SU}(2)_{L}$ doublets and singlets. Given the model predictions and the fit result, we calculate a $\chi^{2}$ value and treat the constraint as fulfilled if $\chi^{2}<\chi_{\text {crit }}^{2}(2 \sigma)$. 


\subsection{Flavour constraints}

Since all of the models currently implemented in ScannerS are naturally flavor conserving [21], the dominant contributions to most flavor observables originate in charged Higgs exchange. Since the charged Higgs sector of all implemented models is either absent or identical to the 2HDM, we treat flavour constraints similar to the EW precision constraints and use the fit results of Ref. [18] providing $2 \sigma$ constraints in the $m_{H^{ \pm}}-\tan \beta$ parameter plane of the 2HDM. These generalize to models with additional singlets, such as the next-to $2 \mathrm{HDM}(\mathrm{N} 2 \mathrm{HDM})^{5}$

\subsection{Higgs searches and Higgs measurements}

Searches for additional scalars as well as measurements of $125 \mathrm{GeV}$ Higgs boson $\left(h_{1} 25\right)$ are among the most important constraints on extended scalar sectors. ScannerS provides an interface to the tools HiggsBounds [22-26] and HiggsSignals $[27,28]$ to incorporate these constraints. The HiggsBounds input can either be given in the effective coupling approximation or by providing all branching ratios and hadronic cross sections to the Higgs bosons. For simple models, such as pure singlet extensions, the effective coupling approximation is exact and used by ScannerS.

Otherwise, ScannerS uses the anyHdecay library (see Appendix A) as an interface to the many adaptations of the code HDECAY [29-31] for different BSM models to obtain model predictions for Higgs boson branching ratios and total widths including state-of-the-art QCD corrections and off-shell effects. To obtain precise cross section predictions, ScannerS includes a tabulated parametrization of the NNLO QCD gluon fusion and $b \bar{b}$-associated Higgs production cross sections at hadron colliders obtained with SusHi1.6.1 [32,33]. Additionally, ScannerS uses cross section parametrizations included in HiggsBounds (see Ref. [26]), such as the $W^{ \pm} / Z$-associated cross sections calculated using VH@NNLO [34,35], as well as the $t$-associated charged Higgs production cross section at NLO QCD [36-41].

HiggsBounds uses this input to check the model predictions against exclusion bounds from Higgs searches at LEP, TEVATRON and the LHC@. Using the expected limit information, only the most sensitive search for each scalar in the model is applied to obtain an approximate combined $2 \sigma$ constraint on the model parameter space. HiggsBounds uses this treatment since applying all of the hundreds of implemented exclusion limits simultaneously would lead to a con-

\footnotetext{
5 Only the $B \rightarrow \mu \mu$ observables receive relevant neutral Higgs contributions, which were left to float in the fit of Ref. [18]. The resulting limit is conservative with respect to the possible impact of the neutral Higgs bosons and can be approximately generalized to models with additional neutral particles.
}

siderably stronger exclusion than the desired $95 \%$ CL. See Refs. [24,26] for details.

HiggsSignals uses the same input as HiggsBounds to calculate a $\chi^{2}$ value that quantifies the agreement of the model prediction with up-to-date measurements of the $h_{1} 25$ properties at the LHC@. In interpreting this $\chi^{2}$, ScannerS uses a profiled likelihood ratio test with the SM as the alternative hypothesis. In the Gaussian approximation the test statistic is

$\Delta \chi^{2}=\chi_{\text {Model }}^{2}-\chi_{\text {SM }}^{2}$,

where both $\chi_{\text {Model }}^{2}$ and $\chi_{\mathrm{SM}}^{2}$ are obtained from HiggsSignals. As discussed in Sect. 1, we use this test statistic - instead of e.g. constructing a goodness-of-fit test using $\chi_{\text {Model }}^{2} /$ d.o.f. - since it allows for a much easier statistical interpretation and does not require knowledge about the best fit point of the model. The resulting $\Delta \chi^{2}$ approximately describes the best-fit region of the parameter space. The upper bound $\chi_{\text {crit }}^{2}$ to be imposed on $\Delta \chi^{2}$ depends on the desired confidence level and on the number $v$ of degrees of freedom. As stated above, we aim to impose $2 \sigma$ constraints in the Gaussian limit and thus $\Delta \chi^{2}<\chi_{\text {crit }}^{2}(2 \sigma, v)$. When presenting results where all but $n$ model parameters and all nuisance parameters are profiled over, the set of allowed parameter points are those with $\Delta \chi^{2}<\chi_{\text {crit }}^{2}(2 \sigma, n)$. In ScannerS, we by default use

$\Delta \chi^{2}<6.18$,

which corresponds to a $2 \sigma$ constraint for $n=2$ under the assumption of Gaussian errors. The choice of a fixed $n=2$ is an arbitrary but very commonly made approximation. It is fully correct in the common case where results are presented as a function of two parameters of interest, i.e. in benchmark planes or scatter plots. In particular, it avoids the issue of correctly determining the appropriate value of $v$, which is challenging and requires detailed knowledge about the best fit point of the model. See Ref. [28] for an in depth discussion of the different possibilities to interpret the $\chi^{2}$ value.

\subsection{Electric dipole moments}

In CP-violating models the stringent limits on fermionic electric dipole moments (EDMs) have to be considered. ScannerS includes a constraint to check the model prediction for the electron EDM against the latest limits by the ACME collaboration [42]. Constraints from the neutron or nuclear EDMs could easily be included in ScannerS if theoretical predictions for these quantities are available in the model.

\subsection{DM constraints}

A global symmetry of an extended scalar sector that remains unbroken after EW symmetry breaking (EWSB) may lead 
to a dark sector with a stable lightest particle. Such a particle is a dark matter (DM) candidate and constraints from DM searches need to be considered. ScannerS includes an interface to the code MicrOMEGAs [43-49] to calculate DM observables. The relic density $\omega_{c}^{\text {model }}$ predicted by the model is required not to exceed the observed dark matter density $\omega_{c}[50]$,

$\omega_{c}^{\text {model }} \leq \omega_{c}+2 \Delta \omega_{c}$,

where $\Delta \omega_{c}$ is the uncertainty of the measurement. By imposing only an upper bound we allow for additional contributions to DM beyond the one stemming from the model under consideration.

ScannerS also imposes constraints from dark matter searches using direct detection - more specifically the XENON1T results [51]. The required dark-matternucleon scattering cross sections are also obtained from MicrOMEGAs. ScannerS currently does not include constraints from indirect detection or collider searches for dark particles, as these are not available as simple, modelindependent limits and require additional effort to interpret them correctly. Since MicrOMEGAs can compute and test some of these constraints, they could be included in ScannerS by extending the existing interface. However, in the parameter scan approach it is also valid to apply additional constraints such as these on top of the ScannerS results using dedicated external computations.

\subsection{A first order EW phase transition}

When studying models that could facilitate EW baryogenesis [52-55] or in the context of gravitational wave signatures [56-63] (see also Ref. [64]) it is interesting to look at parameter regions where the EW phase transition (EWPT) is (strongly) first order. ScannerS includes an interface to the BSMPT $[65,66]$ library to calculate the strength of the EWPT in several of the implemented models. When using this constraint, ScannerS requires that the EWPT is of first order and that the EW vacuum at zero temperature is the global minimum of the 1-loop effective potential. This second requirement - which contains the requirement that the 1-loop effective potential is bounded from below - is a prerequisite for the phase transition search and is verified numerically within BSMPT.

This constraint by default only requires the EWPT to be first order without putting any bound on the strength of the phase transition. However, the critical temperature $T_{c}$ and critical vacuum expectation value (vev) $\omega_{c}{ }^{6}$ are stored in the

\footnotetext{
${ }^{6}$ In BSMPT $\omega_{c}$ is defined as the euclidean norm of the field-space distance between the two degenerate vacua at the critical temperature $T_{c}$. Only vevs of fields that carry EW charge - so no singlet vevs are included in $\omega_{c}$, since only those can contribute to EW baryogenesis $[65,66]$.
}

output, such that more stringent requirements - e.g. $\omega_{c}>T_{c}$ for a strong first order EWPT - can be imposed in postprocessing. Note that in general, there is no physical requirement that the EWPT has to be first order. Therefore, you should only enable this constraint if you wish to study physics associated with a first order EWPT.

\section{BSM Models in ScannerS}

ScannerS includes implementations for a variety of BSM models with extended scalar sectors. In this section, we will give short overviews of the implemented models mainly meant to establish the conventions used in the ScannerS implementation. Additional technical information on all implemented models can be found in the online documentation for the model classes in the Scanners : :Models namespace. The name of the model class and ScannerS executable for each model is indicated in the title of the subsection with the corresponding model or phase description.

\subsubsection{Input parameters and mass-ordering}

As the very first step for each parameter point, ScannerS calculates all model parameters from a chosen set of input parameters. ${ }^{7}$ The input parameters are chosen to allow for a physically motivated selection of scan ranges. This means that physical masses, mixing angles or couplings, and vevs are used rather than directly using the parameters of the scalar potential.

In most BSM models, scalars of identical charge and $\mathrm{CP}, h_{i}$, are distinguished based on their mass - typically by imposing some kind of mass ordering, e.g. $m_{h_{i}} \leq m_{h_{j}}$ for $i<j$. In contrast to more complex BSM models such as supersymmetry or composite Higgs models - the $m_{h_{i}}$ can be chosen as input parameters in all of the scalar extensions implemented in ScannerS. This greatly facilitates phenomenological studies, as one of the Higgs masses can be set to match the observed mass of $h_{1} 25$. However, since the other scalars can in general be either lighter or heavier than $h_{1} 25$ it is inconvenient to use mass ordered states as input. Instead, ScannerS uses a basis of input states $h_{a, b, c, \ldots}$ for which no ordering is required.

As an example, consider a model with three mixing scalars. The three by three mixing matrix is parametrized as

\footnotetext{
${ }^{7}$ In the old ScannerS-1 this calculation was performed numerically and referred to as the generation of a local minimum. Now, the analytic relations between input parameters and the remaining model parameters - e.g. between masses and mixing angles on the one hand and the parameters of the scalar potential on the other - are implemented in closed form, whenever possible.
} 
$R=\left(\begin{array}{ccc}c_{1} c_{2} & s_{1} c_{2} & s_{2} \\ -\left(c_{1} s_{2} s_{3}+s_{1} c_{3}\right) & c_{1} c_{3}-s_{1} s_{2} s_{3} & c_{2} s_{3} \\ -c_{1} s_{2} c_{3}+s_{1} s_{3} & -\left(c_{1} s_{3}+s_{1} s_{2} c_{3}\right) & c_{2} c_{3}\end{array}\right)$,

where $c_{1,2,3}=\cos \alpha_{1,2,3}$ and $s_{1,2,3}=\sin \alpha_{1,2,3}$ for the three mixing angles $-\pi / 2 \leq \alpha_{1,2,3}<\pi / 2$. This parametrization is sufficiently general for physics purposes, but is not a full parametrization of $O(3)$, in particular - since $\cos \alpha_{i} \geq 0$ for the chosen angular ranges - it requires

$\operatorname{det} R=+1, \quad R_{11} \geq 0, \quad R_{33} \geq 0$.

The model input is given in the form of three Higgs masses, e.g.

$m_{h_{a}}=125 \mathrm{GeV}, \quad m_{h_{b}}=300 \mathrm{GeV}, \quad m_{h_{c}}=50 \mathrm{GeV}$,

and values for the three input mixing angles $\alpha_{1,2,3}^{\text {in }}$ that parametrize $R^{\text {in }}$ in the basis

$$
\left(\begin{array}{l}
h_{a} \\
h_{b} \\
h_{c}
\end{array}\right)=R^{\text {in }}\left(\begin{array}{l}
\phi_{1} \\
\phi_{2} \\
\phi_{3}
\end{array}\right)
$$

where $\phi_{1,2,3}$ are the mixing fields of the scalar potential. To convert this to the desired basis $\left(h_{1}, h_{2}, h_{3}\right)^{\mathrm{T}}$ with $m_{h_{1}}<$ $m_{h_{2}}<m_{h_{3}}$, a set of row transpositions is applied on the mixing matrix, such that - for the example mass values of Eq. (7)

$$
\left(\begin{array}{l}
h_{1} \\
h_{2} \\
h_{3}
\end{array}\right) \equiv\left(\begin{array}{l}
h_{c} \\
h_{a} \\
h_{b}
\end{array}\right)=\left(\begin{array}{lll}
R_{c 1}^{\text {in }} & R_{c 2}^{\text {in }} & R_{c 3}^{\text {in }} \\
R_{a 1}^{\text {in }} & R_{a 2}^{\text {in }} & R_{a 3}^{\text {in }} \\
R_{b 1}^{\text {in }} & R_{b 2}^{\text {in }} & R_{b 3}^{\text {in }}
\end{array}\right)\left(\begin{array}{l}
\phi_{1} \\
\phi_{2} \\
\phi_{3}
\end{array}\right) .
$$

In general, the resulting mixing matrix no longer fulfills Eq. (6) and is thus no longer parametrized by Eq. (5). The parametrization can be restored by physically irrelevant field redefinitions of the form $h_{i} \rightarrow-h_{i}$ that flip the sign of all elements in a row of the mixing matrix, i.e. $h_{1} \rightarrow-h_{1}$ if $R_{11}<0, h_{3} \rightarrow-h_{3}$ if $R_{33}<0$, and finally $h_{2} \rightarrow-h_{2}$ if $\operatorname{det} R=-1.8$

This procedure works analogously for other dimensionalities or different parametrizations of $R$. It can also be used if not all of the masses are input parameters. In this case the remaining masses are first calculated using $R^{\text {in }}$ and then the reordering is performed.

The input parametrizations in ScannerS are usually agnostic regarding which of the $H_{a}$ is identified with $h_{1} 25$. Any exceptions to this are stated in the description of the parametrizations below.

\footnotetext{
8 The corresponding implementation can be found in Scanners : :Utilities : : OrderedMixMat3d.
}

\subsection{The complex-singlet-extension of the SM}

Pure extension of the SM by gauge-singlet scalar fields are the simplest possible extended scalar sectors. The complexsinglet-extension of the SM (CxSM) $[1,67,68]$ adds a complex singlet field $\mathbb{S}$ with a softly broken $U(1)$ symmetry to the SM. The implementation in ScannerS follows Refs. [1,68] where an additional symmetry under $\mathbb{S} \rightarrow \mathbb{S}^{*}$ is imposed that forces all model parameters to be real. The resulting scalar potential is

$$
\begin{aligned}
V_{\mathrm{CxSM}}= & \frac{m^{2}}{2} \Phi^{\dagger} \Phi+\frac{\lambda}{4}\left(\Phi^{\dagger} \Phi\right)^{2}+\frac{\delta_{2}}{2} \Phi^{\dagger} \Phi|\mathbb{S}|^{2} \\
& +\frac{b_{2}}{2}|\mathbb{S}|^{2}+\frac{d_{2}}{4}|\mathbb{S}|^{4} \\
& +\left(\frac{b_{1}}{4} \mathbb{S}^{2}+a_{1} \mathbb{S}+\text { h.c. }\right)
\end{aligned}
$$

with seven real parameters. The CxSM allows for different phases, where different fields acquire vevs. Implementations for the broken phase and the dark phase are included in ScannerS. The following constraints are included for both phases with very similar implementations:

- The perturbative unitarity constraint was obtained purely numerically in Refs. [1,68]. ScannerS instead uses the analytic conditions (see Appendix B)

$$
\begin{aligned}
& \frac{|\lambda|}{2}, \frac{\left|\delta_{2}\right|}{2}, \frac{\left|d_{2}\right|}{2}<8 \pi, \\
& \frac{\left|2 d_{2}+3 \lambda \pm \sqrt{8 \delta_{2}^{2}+\left(2 d_{2}-3 \lambda\right)^{2}}\right|}{4}<8 \pi .
\end{aligned}
$$

- Boundedness from below is ensured using the known analytic conditions [1].

- The oblique parameters are calculated and tested using the generic method described in Sect. 2.2.

- The branching ratios of the scalars are calculated using sHDECAY [68] through the anyHdecay interface (see Appendix A).

- Predictions for gluon-fusion and $b b$-associated Higgs production at hadron colliders are obtained using tabulated results from SusHi (see Sect. 2.4) and the parametrization included in HiggsBounds is used to obtain cross sections for the $V H$-associated (sub) channels (see Ref. [26]).

- Constraints from Higgs searches and Higgs measurements are tested with HiggsBounds and HiggsSignals as described in Sect. 2.4 using the aforementioned predictions for branching ratios and cross sections.

- Using BSMPT for the calculation, the EWPT can be required to be first order. Since this requirement is not 
a necessary constraint it is not enabled by default (see Sect. 2.7).

\subsubsection{The broken-phase CXSM-CXSMBroken}

The CxSM is in the broken phase if both the real and imaginary parts of $\mathbb{S}$ acquire non-zero vevs. This leads to a Higgs sector with three mixing CP-even scalar bosons $h_{i}$ $(i \in\{1,2,3\})$. ScannerS uses the input parameters [68]

$m_{a}, \quad m_{b}, \quad \alpha_{1}^{\text {in }}, \quad \alpha_{2}^{\text {in }}, \quad \alpha_{3}^{\text {in }}, \quad v_{S}, \quad v=v_{\mathrm{EW}}$,

where $m_{a, b}$ are the masses of two of the $h_{i}, \alpha_{1,2,3}^{\text {in }}$ are the input mixing angles, $v_{S}$ is the real singlet vev, and $v_{\mathrm{EW}} \approx 246 \mathrm{GeV}$ is the EW vev. The third scalar input mass $m_{c}$ is calculated through

$m_{c}^{2}=-\frac{m_{a}^{2} m_{b}^{2} R_{c 1}^{\mathrm{in}} R_{c 2}^{\mathrm{in}}}{m_{a}^{2} R_{b 1}^{\mathrm{in}} R_{b 2}^{\mathrm{in}}+m_{b}^{2} R_{a 1}^{\text {in }} R_{a 2}^{\text {in }}}$.

If Eq. (13) predicts a tachyonic $m_{c}^{2}$ the corresponding parameter point is rejected.

\subsubsection{The dark-phase CXSM-CXSMDark}

If the imaginary part of $\mathbb{S}$ acquires no vev during EWSB, the corresponding particle is a dark-matter candidate stabilized by a $\mathbb{Z}_{2}$ symmetry. The real component of the singlet field still has a non-zero vev and mixes with the SM Higgs boson. The additional assumption $a_{1}=0$ is made, such that this dark phase of the CXSM only has six real parameters:

$m_{a}, \quad m_{b}, \quad m_{X}, \quad \alpha^{\text {in }} \quad v_{S}, \quad v=v_{\mathrm{EW}}$,

where $m_{a, b}$ are the masses of the two visible Higgs bosons, $m_{X}$ is the mass of the dark scalar, $\alpha^{\text {in }}$ is the input mixing angle, and $v_{S}$ is the real singlet vev. The convention for the mixing angle $\alpha$ matches Eq. (5) with $\alpha_{1} \rightarrow \alpha$ and $\alpha_{2,3} \rightarrow 0$ (and analogously for the $\alpha^{\text {in }}$ ). Since the dark phase contains a DM candidate, the corresponding DM constraints have to be considered in addition to the constraints discussed in Sect. 3.1:

- Dark matter observables are calculated using MicrOMEGAs and tested against the experimental limits as discussed in Sect. 2.6.

\subsection{The two-real-singlet-extension of the SM}

The two-real-singlet-extension of the SM (TRSM) [69] is a different extension of the SM by two real scalar degrees of freedom. The scalar potential in terms of two real scalar fields
$S$ and $X$ is given by

$$
\begin{aligned}
V_{\mathrm{TRSM}}= & \mu_{\Phi}^{2} \Phi^{\dagger} \Phi+\lambda_{\Phi}\left(\Phi^{\dagger} \Phi\right)^{2}+\mu_{S}^{2} S^{2} \\
& +\lambda_{S} S^{4}+\mu_{X}^{2} X^{2}+\lambda_{X} X^{4}+\lambda_{\Phi S} \Phi^{\dagger} \Phi S^{2} \\
& +\lambda_{\Phi X} \Phi^{\dagger} \Phi X^{2}+\lambda_{X S} S^{2} X^{2}
\end{aligned}
$$

This scalar potential respects a $\mathbb{Z}_{2} \otimes \mathbb{Z}_{2}$ symmetry for $S$ and $X$ and has nine real parameters. Depending on the vacuum structure the TRSM allows for different phases, though only the broken phase is currently implemented.

\subsubsection{The broken-phase TRSM - TRSMBroken}

In the broken phase both $S$ and $X$ acquire vacuum expectation values $v_{S}$ and $v_{X}$, respectively. This phase with three mixing CP-even scalars $h_{i}(i \in\{1,2,3\})$ was studied in detail in Ref. [69] and we follow the conventions used there. The input parameters are

$M_{a}, \quad M_{b}, \quad M_{c}, \quad \theta_{h S}^{\text {in }} \theta_{h X}^{\text {in }}, \quad \theta_{S X}^{\text {in }}, \quad v_{S}, \quad v_{X}, \quad v=v_{\mathrm{EW}}$,

where $M_{a, b, c}$ are the three Higgs masses, $\theta_{h S}^{\text {in }}, \theta_{h X}^{\text {in }}, \theta_{S X}^{\text {in }}$ are the input mixing angles, and $v_{S, X}$ are the singlet vevs. The mixing angles $\theta$ parametrize the mixing matrix $R$ of Eq. (5) as

$\theta_{h S} \equiv-\alpha_{1}, \quad \theta_{h X} \equiv-\alpha_{2}, \quad \theta_{S X} \equiv-\alpha_{3}$.

Analogous relations hold for the corresponding input quantities $\theta^{\text {in }}, R^{\text {in }}$, and $\alpha^{\text {in }}$.

In the broken phase of the TRSM, ScannerS implements the following constraints and calculations:

- Perturbative unitarity and boundedness from below are ensured using the analytic conditions given in Ref. [69].

- The oblique parameters are calculated and tested using the generic method of Sect. 2.2.

- The Higgs-to-Higgs decay widths of the scalars are calculated at tree-level and combined with the appropriately rescaled SM-like branching ratios as tabulated in HiggsBounds using the effective coupling input (see Ref. [26]).

- HiggsBounds and HiggsSignals are used to test constraints from Higgs data as described in Sect. 2.4.

\subsection{The two-Higgs-doublet model}

The 2HDM [70] (see e.g. Ref. [71] for a review) is probably the most studied non-supersymmetric scalar extension of the 
SM. In the conventions of Ref. [71] the scalar potential of the $2 \mathrm{HDM}$ with a softly broken $\mathbb{Z}_{2}$ symmetry is given by

$$
\begin{aligned}
V_{2 \mathrm{HDM}}= & m_{11}^{2} \Phi_{1}^{\dagger} \Phi_{1}+m_{22}^{2} \Phi_{2}^{\dagger} \Phi_{2}-\left(m_{12}^{2} \Phi_{1}^{\dagger} \Phi_{2}+\text { h.c. }\right) \\
& +\frac{\lambda_{1}}{2}\left(\Phi_{1}^{\dagger} \Phi_{1}\right)^{2}+\frac{\lambda_{2}}{2}\left(\Phi_{2}^{\dagger} \Phi_{2}\right)^{2}+\lambda_{3}\left(\Phi_{1}^{\dagger} \Phi_{1}\right)\left(\Phi_{2}^{\dagger} \Phi_{2}\right) \\
& +\lambda_{4}\left(\Phi_{1}^{\dagger} \Phi_{2}\right)\left(\Phi_{2}^{\dagger} \Phi_{1}\right)+\left(\frac{\lambda_{5}}{2}\left(\Phi_{1}^{\dagger} \Phi_{2}\right)^{2}+\text { h.c. }\right)
\end{aligned}
$$

The softly broken $\mathbb{Z}_{2}$ symmetry is extended to the Yukawa sector to prevent tree-level flavour changing neutral currents leading to the four Yukawa types of the 2HDM.

The parameters $m_{12}^{2}$ and $\lambda_{5}$ of Eq. (18) can take complex values and lead to a $\mathrm{CP}$-violating scalar sector. ScannerS implements two variants of the $2 \mathrm{HDM}$ - the CP-conserving real 2HDM (R2HDM) and the CP-violating complex 2HDM (C2HDM) [72-74]. The following constraints have similar implementations in both cases.

- Perturbative unitarity and boundedness from below are tested using the analytic conditions given in Ref. [71].

- The oblique parameters are calculated and tested using the generic method of Sect. 2.2.

- Constraints from $b$-physics are tested as discussed in Sect. 2.3.

- Using BSMPT for the calculation, the EWPT can be required to be first order. Since this requirement is not a necessary constraint it is not enabled by default (see Sect. 2.7).

\subsubsection{The R2HDM - R2HDM}

In the R2HDM, CP-conservation is imposed in the Higgs sector forcing all eight parameters of Eq. (18) to be real. In the R2HDM, the mixing matrix is conventionally defined through

$$
\left(\begin{array}{l}
H \\
h
\end{array}\right)=\left(\begin{array}{cc}
\cos \alpha & \sin \alpha \\
-\sin \alpha & \cos \alpha
\end{array}\right)\left(\begin{array}{l}
\rho_{1} \\
\rho_{2}
\end{array}\right) \quad \text { with }-\pi / 2 \leq \alpha<\pi / 2,
$$

where $\rho_{1,2}$ are the real, neutral component fields of the two doublets. One set of input parameters implemented in ScannerS is thus

$$
\begin{array}{llll}
m_{H_{a}}, & m_{H_{b}}, & m_{A}, & m_{H^{ \pm}}, \quad \alpha^{\text {in }}, \\
\tan \beta, & m_{12}^{2}, & v=v_{\mathrm{EW}}, &
\end{array}
$$

where $m_{H_{a, b}}$ are the neutral, CP-even Higgs masses, $m_{A}$ is the mass of the pseudoscalar, $m_{H}^{ \pm}$is the charged Higgs mass, $\alpha^{\text {in }}$ is the CP-even neutral sector input mixing angle, $\tan \beta=$ $v_{2} / v_{1}$ is the ratio of the vevs, and $m_{12}^{2}$ is the soft $\mathbb{Z}_{2}$-breaking parameter. Furthermore, the Yukawa type has to be specified, which can be either

$$
\begin{array}{ll}
1 \equiv \text { type I }, & 2 \equiv \text { type II } \\
3 \equiv \text { lepton specific }, & 4 \equiv \text { flipped }
\end{array}
$$

Instead of using $\alpha^{\text {in }}$ as input parameter, ScannerS also implements an alternative (default) input parametrization in terms of the effective gauge coupling of $H_{b}$,

$c\left(H_{b} V V\right)=\sin \left(\beta-\alpha^{\text {in }}\right)$.

Restricting $c\left(H_{b} V V\right) \sim 0$ forces $H_{a}$ to be close to the alignment limit. This is the default parametrization of the R2HDM consisting of

$$
\begin{aligned}
& m_{H_{a}}, \quad m_{H_{b}}, \quad m_{A}, \quad m_{H^{ \pm}}, \quad c\left(H_{b} V V\right), \\
& \tan \beta, \quad m_{12}^{2}, \quad v=v_{\mathrm{EW}} \text {, }
\end{aligned}
$$

together with the Yukawa type.

The following constraints and calculations in the R2HDM are implemented in addition to the ones mentioned in Sect. 3.3:

- Absolute stability of the EW vacuum is ensured using the discriminant of Ref. [75].

- The branching ratios of the scalars are calculated using HDECAY [29-31] through the anyHdecay interface (see Appendix A).

- Predictions for gluon-fusion and $b b$-associated Higgs production at hadron colliders are obtained using tabulated results from SUSHI (see Sect. 2.4). The HiggsBounds parametrizations are used to obtain cross section predictions for the $V H$-associated (sub)channels and for charged Higgs production in association with a top-quark (see Ref. [26]).

- HiggsBounds and HiggsSignals are used to test constraints from Higgs data as described in Sect. 2.4.

\subsubsection{The C2HDM-C2HDM}

If complex values for $m_{12}^{2}$ and $\lambda_{5}$ are allowed, the 2HDM scalar potential can be CP-violating [73]. The phases of these two parameters are not both independent, such that the $\mathrm{C} 2 \mathrm{HDM}$ has nine real free parameters. The $\mathrm{C} 2 \mathrm{HDM}$ was discussed in detail in Ref. [76] and we follow the conventions used there. The $3 \times 3$ mixing matrix of the neutral scalars is parametrized as in Eq. (5). The input parametrization in terms of the mixing angles $\alpha_{1,2,3}^{\text {in }}$ is

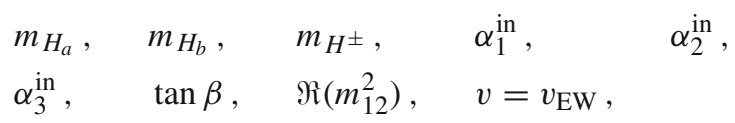


where $m_{H_{a, b}}$ are two of the neutral Higgs masses, $m_{H^{ \pm}}$is the charged Higgs mass, $\alpha_{1,2,3}^{\text {in }}$ are the neutral-sector input mixing angles, $\tan \beta=v_{2} / v_{1}$ is the ratio of the vevs and $\Re\left(m_{12}^{2}\right)$ is the real part of the soft $\mathbb{Z}_{2}$-breaking parameter. Furthermore, the Yukawa type needs to be specified. The third neutral scalar mass $m_{H_{c}}$ is calculated from the two other ones using [76]

$$
\begin{aligned}
& m_{H_{c}}^{2} \\
& =\frac{m_{H_{a}}^{2} R_{a 3}^{\text {in }}\left(R_{a 2}^{\text {in }} \tan \beta-R_{a 1}^{\text {in }}\right)+m_{H_{b}}^{2} R_{b 3}^{\text {in }}\left(R_{b 2}^{\text {in }} \tan \beta-R_{b 1}^{\text {in }}\right)}{R_{c 3}^{\text {in }}\left(R_{c 1}^{\text {in }}-R_{c 2}^{\text {in }} \tan \beta\right)} .
\end{aligned}
$$

If this results in a tachyonic $H_{c}$ the parameter point is rejected.

ScannerS implements a second C2HDM input parametrization in terms of the couplings and mixing matrix elements of $H_{a}$ and $H_{b}$. The input parameters are

$$
\begin{aligned}
& m_{H_{a}}, \quad m_{H_{b}}, \quad m_{H^{ \pm}}, \\
& \tan \beta, \quad \Re\left(m_{12}^{2}\right), \quad v=v_{\mathrm{EW}}, \\
& c^{2}\left(H_{a} V V\right), \quad\left|c\left(H_{a} t \bar{t}\right)\right|^{2}, \quad \operatorname{sign}\left(R_{a 3}^{\text {in }}\right), \quad R_{b 3}^{\text {in }},
\end{aligned}
$$

where $c\left(H_{a} V V\right)$ denotes the effective coupling of $H_{a}$ to the massive gauge bosons $\left(V \in\left\{W^{ \pm}, Z\right\}\right),\left|c\left(H_{a} t \bar{t}\right)\right|^{2}$ is the squared absolute value of the effective coupling between $H_{a}$ and top-quarks, and $R_{a 3}^{\text {in }}$ and $R_{b 3}^{\text {in }}$ are the mixing matrix elements between the input states $H_{a, b}$ and the pseudoscalar gauge eigenstate. Additionally, the Yukawa type has to be specified. The second sign required to compensate for the two squared couplings as input is fixed by assuming

$c\left(H_{a} V V\right) c^{e}\left(H_{a} t \bar{t}\right)>0$.

This assumption is enforced by the Higgs measurements for $H_{a} \equiv h_{125}$ which should be chosen when using this parametrization. The mixing angles are obtained from these couplings using the relations

$$
\begin{aligned}
c\left(H_{a} V V\right) & =\cos \beta R_{a 1}^{\mathrm{in}}+\sin \beta R_{a 2}^{\mathrm{in}}, \\
\left|c\left(H_{a} t \bar{t}\right)\right|^{2} & =\left(c^{e}\left(H_{a} t \bar{t}\right)\right)^{2}+\left(c^{o}\left(H_{a} t \bar{t}\right)\right)^{2} \\
& =\left(\frac{R_{a 2}}{\sin \beta}\right)^{2}+\left(\frac{R_{a 3}}{\tan \beta}\right)^{2},
\end{aligned}
$$

in combination with Eq. (5), where $c^{e}$ and $c^{o}$ refer to the CPeven and CP-odd components of the effective Higgs-fermion coupling, respectively. If this system of conditions does not yield a valid solution for $\alpha_{1,2,3}^{\text {in }}$ the parameter point is rejected. This parametrization is very useful to ensure that $H_{a} \equiv h_{125}$ has SM-like couplings and is used by default.

ScannerS checks the following constraints in addition to the ones mentioned in Sect. 3.3:
- Absolute stability of the EW vacuum is ensured using the discriminant of Ref. [77].

- The branching ratios of the scalars are calculated using C2HDM_HDECAY [76] through the anyHdecay interface (see Appendix A).

- Predictions for gluon-fusion and $b b$-associated Higgs production at hadron colliders are obtained using tabulated results from SUSHI (see Sect. 2.4). CP-mixing effects are included, but CP-interference effects like the ones discussed in [78] are not included. The HiggsBounds parametrizations are used to obtain cross section predictions for the CP-mixed $V H$-associated (sub)channels and for charged Higgs production in association with a top-quark (see Ref. [26]).

- HiggsBounds and HiggsSignals are used to test constraints from Higgs data as described in Sect. 2.4.

- The model prediction for the electron EDM is calculated following Ref. [79] and checked against the limit by the ACME collaboration [42].

\subsection{The next-to $2 \mathrm{HDM}$}

The next-to 2HDM (N2HDM) [80-82] adds an additional real scalar singlet field $\Phi_{S}$ to the R2HDM. In the conventions of Ref. [82] its scalar potential is given by

$$
\begin{aligned}
V_{\mathrm{N} 2 \mathrm{HDM}}= & m_{11}^{2} \Phi_{1}^{\dagger} \Phi_{1}+m_{22}^{2} \Phi_{2}^{\dagger} \Phi_{2}-m_{12}^{2}\left(\Phi_{1}^{\dagger} \Phi_{2}+\text { h.c. }\right) \\
& +\frac{\lambda_{1}}{2}\left(\Phi_{1}^{\dagger} \Phi_{1}\right)^{2}+\frac{\lambda_{2}}{2}\left(\Phi_{2}^{\dagger} \Phi_{2}\right)^{2}+\lambda_{3}\left(\Phi_{1}^{\dagger} \Phi_{1}\right)\left(\Phi_{2}^{\dagger} \Phi_{2}\right) \\
& +\lambda_{4}\left(\Phi_{1}^{\dagger} \Phi_{2}\right)\left(\Phi_{2}^{\dagger} \Phi_{1}\right) \\
& +\frac{\lambda_{5}}{2}\left(\left(\Phi_{1}^{\dagger} \Phi_{2}\right)^{2}+\text { h.c. }\right)+\frac{m_{S}^{2}}{2} \Phi_{S}^{2}+\frac{\lambda_{6}}{8} \Phi_{S}^{4} \\
& +\frac{\lambda_{7}}{2}\left(\Phi_{1}^{\dagger} \Phi_{1}\right) \Phi_{S}^{2}+\frac{\lambda_{8}}{2}\left(\Phi_{2}^{\dagger} \Phi_{2}\right) \Phi_{S}^{2} .
\end{aligned}
$$

The N2HDM scalar potential has an exact $\mathbb{Z}_{2}$ symmetry for the singlet field in addition to the softly broken $\mathbb{Z}_{2}$ symmetry of the doublet field inherited from the 2HDM. The doublet $\mathbb{Z}_{2}$ symmetry leads to the same $2 \mathrm{HDM}$ types when extended to the Yukawa sector. The N2HDM features different phases, depending on which symmetries remain unbroken after EWSB. Most constraints and calculations are implemented similarly for all phases of the N2HDM:

- Perturbative unitarity and boundedness from below [83] are ensured using the analytic conditions given in Ref. [82].

- The oblique parameters are calculated and tested using the generic method of Sect. 2.2.

- The branching ratios of the scalars are calculated using N2HDECAY $[82,84]$ through the anyHdecay interface (see Appendix A). 
- Predictions for gluon-fusion and $b b$-associated Higgs production at hadron colliders are obtained using tabulated results from SUSHI (see Sect. 2.4). The HiggsBounds parametrizations are used to obtain cross section predictions for the $V H$-associated (sub)channels and for charged Higgs production in association with a top-quark (see Ref. [26]).

- HiggsBounds and HiggsSignals are used to test constraints from Higgs data as described in Sect. 2.4.

- Metastability constraints on the stability of the EW vacuum are obtained using the EVADE library. See also Ref. [15] for a detailed study of vacuum stability in the brokenphase N2HDM.

\subsubsection{The broken-phase N2HDM - N2HDMBroken}

If both doublets and the singlet fields acquire non-zero vevs the EW vacuum of the N2HDM is in the broken phase. In this phase, the three CP-even neutral scalar fields mix with the $3 \times 3$ mixing matrix parametrized as Eq. (5). The input parametrization in terms of the mixing angles $\alpha_{1,2,3}^{\text {in }}$ is

$$
\begin{array}{llll}
m_{H_{a}}, & m_{H_{b}}, & m_{H_{c}}, & \\
m_{A}, & m_{H^{ \pm}}, & \tan \beta, & \alpha_{1}^{\text {in }}, \alpha_{2}^{\text {in }}, \\
\alpha_{3}^{\text {in }}, & m_{12}^{2}, & v_{S}, & v=v_{\mathrm{EW}},
\end{array}
$$

where $m_{H_{a, b, c}}$ are the CP-even scalar Higgs masses, $m_{A}$ is the pseudoscalar mass, $m_{H^{ \pm}}$is the charged Higgs mass, $\tan \beta=v_{2} / v_{1}$ is the ratio of the doublet vevs, $\alpha_{1,2,3}^{\text {in }}$ are the input mixing angles of the $\mathrm{CP}$-even neutral scalar sector, $m_{12}^{2}$ is the soft $\mathbb{Z}_{2}$-breaking parameter and $v_{S}$ is the singlet vev. Additionally, the Yukawa type has to be specified as in Eq. (21). Similar to the C2HDM we provide a reparametrization in terms of effective couplings and mixing matrix elements. In this case the input parameters are

$$
\begin{array}{lll}
m_{H_{a}}, & m_{H_{b}}, & m_{H_{c}}, \\
m_{A}, & m_{H^{ \pm}}, & \tan \beta, \\
c^{2}\left(H_{a} V V\right), & c^{2}\left(H_{a} t \bar{t}\right), & \operatorname{sign}\left(R_{a 3}^{\text {in }}\right), \quad R_{b 3}^{\text {in }} \\
m_{12}^{2}, & v_{S}, & v=v_{\mathrm{EW}},
\end{array}
$$

where $c\left(H_{a} V V\right)$ and $c\left(H_{a} t \bar{t}\right)$ are the effective couplings of $H_{a}$ to massive gauge bosons and top-quarks, respectively, while $R_{a 3}^{\text {in }}$ and $R_{b 3}^{\text {in }}$ are the mixing matrix elements between $H_{a, b}$ and the singlet field. The Yukawa type has to be specified as an additional input parameter. The second sign required to compensate for the squared coupling input is again fixed by the assumption

$c\left(H_{a} V V\right) c\left(H_{a} t \bar{t}\right)>0$,

which is physically motivated for $H_{a} \equiv h_{125}$ which should be chosen when using this parametrization. We obtain the mixing angles from the couplings using

$$
\begin{aligned}
c\left(H_{a} V V\right) & =\cos \beta R_{a 1}^{\text {in }}+\sin \beta R_{a 2}^{\text {in }}, \\
c\left(H_{a} t \bar{t}\right) & =\frac{R_{a 2}^{\text {in }}}{\sin \beta},
\end{aligned}
$$

and Eq. (5).

On top of the constraints listed in Sect. 3.4, the broken phase N2HDM implements the following:

- Using BSMPT for the calculation [85], the EWPT can be required to be first order. Since this requirement is not a necessary constraint it is not enabled by default (see Sect. 2.7).

\subsubsection{The dark-singlet-phase N2HDM - N2HDMDarkS}

If the singlet vev is zero after EWSB, the singlet field $\Phi_{S}$ is a mass eigenstate stabilized by the $\mathbb{Z}_{2}$ symmetry of $\Phi_{S}$. In this dark-singlet-phase [81,84,86,87] we follow the conventions of Ref. [87]. The mixing between the two visible CP-even neutral scalars is treated in the convention of the R2HDM in Eq. (19), though - for better analogy to the other phases this $2 \times 2$ mixing is embedded in a $3 \times 3$ mixing matrix [87]. The input parameters are

$$
\begin{array}{llllll}
m_{H_{a}}, & m_{H_{b}}, & m_{A}, & m_{H^{ \pm}}, & m_{H_{D}}, & \tan \beta, \\
\alpha^{\text {in }}, & m_{12}^{2}, & \lambda_{6}, & \lambda_{7}, & \lambda_{8}, & v=v_{\mathrm{EW}},
\end{array}
$$

together with the Yukawa type, where now $m_{H_{a, b}}$ are the masses of the visible CP-even scalars, $m_{H_{D}}$ is the mass of the dark scalar, and $\alpha^{\text {in }}$ is the remaining visible-sector mixing angle. In addition to the constraints listed in Sect. 3.4, the dark-singlet-phase N2HDM also implements the following:

- Dark matter observables are calculated using MicrOMEGAs and tested against the experimental limits as discussed in Sect. 2.6. Due to the limitations of the model file format used by MicrOMEGAs, dark matter observables can currently only be calculated for Yukawa sectors of type I.

\subsubsection{The dark-doublet-phase N2HDM - N2HDMDarkD}

If $m_{12}^{2}=0$ it is possible that only one Higgs doublet of the N2HDM acquires a vev. The resulting exact $\mathbb{Z}_{2}$ symmetry leads to an inert Higgs doublet similar to the inert doublet model [88]. As long as the singlet vev is non-zero, there is still mixing between the SM-like doublet Higgs and the singlet. We again follow the conventions of Ref. [87]. The ScannerS 
input parameters in the dark-doublet-phase N2HDM are

$\begin{array}{lllll}m_{H_{a}}, & m_{H_{b}}, & m_{A_{D}}, & m_{H_{D}^{ \pm}}, & m_{H_{D}}, \quad \alpha^{\text {in }}, \\ m_{22}^{2}, & \lambda_{2}, & \lambda_{8}, & v_{S}, & v=v_{\mathrm{EW}},\end{array}$

where $m_{A_{D}}$ and $m_{H^{D}}$ denote the masses of the two oppositeCP neutral dark scalars and $m_{H_{D}^{ \pm}}$is the dark charged scalar mass. Since one of the doublets is inert, there are no Yukawa types in this phase. The dark-doublet-phase N2HDM implements the following additional constraints:

- Dark matter observables are calculated using MicrOMEGAs and tested against the experimental limits as discussed in Sect. 2.6.

\subsubsection{The fully-dark-phase N2HDM - N2HDMDarkSD}

If the singlet vev additionally vanishes, both $\mathbb{Z}_{2}$ symmetries of the N2HDM remain exact after EWSB. This phase features a visible sector identical to the SM and two distinct dark sectors - one containing the inert doublet and the other composed of just the singlet - that are also stabilized with respect to each other. Since there is no mixing in this phase, the input parametrization is extremely simple. In the conventions of Ref. [87], ScannerS uses the parameters

$$
\begin{aligned}
& m_{H_{\mathrm{SM}}}, \quad m_{H_{D}^{D}}, \quad m_{A_{D}}, \quad m_{H_{D}^{ \pm}}, \quad m_{H_{D}^{S}}, \quad m_{22}^{2}, \\
& m_{S}^{2} \quad \lambda_{2}, \quad \lambda_{6}, \quad \lambda_{8}, \quad v=v_{\mathrm{EW}},
\end{aligned}
$$

where $H_{D}^{D}$ is the real, neutral dark Higgs from the inert doublet sector, and $H_{D}^{S}$ is the dark singlet. The fully-dark N2HDM also implements DM constraints:

- Dark matter observables are calculated using MicrOMEGAs and tested against the experimental limits as discussed in Sect. 2.6. The MicrOMEGAs routines adapted for two-component DM are used.

\subsection{Minimal CP-violating dark matter - CPVDM}

The minimal model of CP-violating scalar dark matter [89] is a variant of the N2HDM, where the two separate $\mathbb{Z}_{2}$ symmetries for the doublet and singlet fields are merged into one. The resulting scalar potential in the conventions of Ref. [89] is

$$
\begin{aligned}
V_{\mathrm{CPVDM}}= & m_{11}^{2} \Phi_{1}^{\dagger} \Phi_{1}+m_{22}^{2} \Phi_{2}^{\dagger} \Phi_{2}+\frac{m_{S}^{2}}{2} \Phi_{S}^{2} \\
& +\left(A \Phi_{1}^{\dagger} \Phi_{2} \Phi_{S}+\text { h.c. }\right) \\
& +\frac{\lambda_{1}}{2}\left(\Phi_{1}^{\dagger} \Phi_{1}\right)^{2}+\frac{\lambda_{2}}{2}\left(\Phi_{2}^{\dagger} \Phi_{2}\right)^{2} \\
& +\lambda_{3}\left(\Phi_{1}^{\dagger} \Phi_{1}\right)\left(\Phi_{2}^{\dagger} \Phi_{2}\right)+\lambda_{4}\left(\Phi_{1}^{\dagger} \Phi_{2}\right)\left(\Phi_{2}^{\dagger} \Phi_{1}\right) \\
& +\frac{\lambda_{5}}{2}\left(\left(\Phi_{1}^{\dagger} \Phi_{2}\right)^{2}+\text { h.c. }\right) \\
& +\frac{\lambda_{6}}{8} \Phi_{S}^{4}+\frac{\lambda_{7}}{2}\left(\Phi_{1}^{\dagger} \Phi_{1}\right) \Phi_{S}^{2}+\frac{\lambda_{8}}{2}\left(\Phi_{2}^{\dagger} \Phi_{2}\right) \Phi_{S}^{2},
\end{aligned}
$$

where the trilinear parameter $A$ can be complex. ${ }^{9}$ Only $\Phi_{1}$ acquires a vev such that the visible sector is identical to the $\mathrm{SM}$, but $\mathrm{CP}$-violating mixing between the three neutral fields in the dark sector is induced by the $A$ term. With the mixing matrix parametrized as in Eq. (5) the ScannerS input parameters are

$$
\begin{aligned}
& m_{h}, \quad m_{h_{a}}, \quad m_{h_{b}}, m_{H^{ \pm}}, \quad \alpha_{1}^{\text {in }}, \quad \alpha_{2}^{\text {in }}, \quad \alpha_{3}^{\text {in }}, \\
& \lambda_{2}, \quad \lambda_{6}, \quad \lambda_{8}, \quad m_{22}^{2}, \quad m_{S}^{2}, \quad v=v_{\mathrm{EW}} \text {, }
\end{aligned}
$$

where $h$ is the SM-like visible Higgs boson and $h_{a, b}$ are dark neutral scalars. The third dark neutral Higgs mass $m_{h_{c}}$ is calculated through the relation

$m_{h_{c}}^{2}=-\frac{m_{h_{a}}^{2} R_{a 1} R_{a 2}+m_{h_{b}}^{2} R_{b 1} R_{b 2}}{R_{c 1} R_{c 2}}$.

The parameter point is rejected if this leads to a tachyonic $h_{c}$. The CP violation in this model does not enter the fermion sector and, therefore, no CP-sensitive EDM constraints need to be considered for the model. The implemented constrains are:

- Perturbative unitarity and boundedness from below are ensured using the same analytic conditions as in the N2HDM [82].

- The oblique parameters are calculated and tested using the generic method of Sect. 2.2.

- The Higgs-to-Higgs decay widths of the scalars are calculated at tree-level and combined with the appropriately rescaled SM-like branching ratios as tabulated in HiggsBounds using the effective coupling input (see Ref. [26]).

- HiggsBounds and HiggsSignals are used to test constraints from Higgs data as described in Sect. 2.4.

- Dark matter observables are calculated using MicrOMEGAs and tested against the experimental limits as discussed in Sect. 2.6.

\footnotetext{
9 A priori, $\lambda_{5}$ could also be complex, but it can always be made real by a rephasing of the fields.
} 
- Metastability constraints on the stability of the EW vacuum are obtained using the EVADE library. See also Ref. [15] for a detailed study of vacuum stability in the brokenphase N2HDM.

\section{User operating instructions}

The ScannerS source code is available at https://gitlab.com/ jonaswittbrodt/ScannerS.

ScannerS requires the following tools and libraries to be available on the system:

- While most of the code is written in $\mathrm{C}++$, some of the dependencies are Fortran codes such that working compilers for $\mathrm{C}, \mathrm{C}++$, and Fortran are required. The $\mathrm{C}++$ compiler has to support at least $\mathrm{C}++-17$.

- ScannerS is compiled using CMake which has to be available on the system.

- The GSL [90] is used for some numerical operations.

- The Eigen3 library [91] is used throughout the code.

Detailed information on the required versions is included in the README file.

If these basic requirements are fulfilled, ScannerS can be compiled using

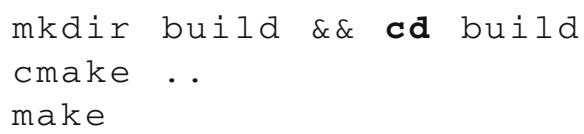

after which the ScannerS test suite can be run with make test. The physics codes required by ScannerS are automatically downloaded by CMake. These currently include HiggsBounds [22-26], HiggsSignals [27,28], and anyHdecay (see Appendix A). Additionally, there are several optional dependencies to enable additional constraints.

- MicrOMEGAs [43-49] is needed for the calculation of DM observables.

- EVADE [14-16] is needed for metastability constraints.

- BSMPT $[65,66]$ is needed to compute the order and strength of the EW phase transition.

Again, see the README file for more technical information on how to link these codes to ScannerS.

Compiling ScannerS generates one executable in the build directory for each implemented model. The executable names for each model are given in the title of the corresponding subsection in Sect. 3. All of the ScannerS executables support two run modes: scan mode and check mode. In scan mode, ScannerS generates and tests random parameter points until the requested number of valid points is found. It can be called as e.g.

$$
\begin{aligned}
. / \text { R2HDM } & - \text {-config । } \\
& \text { example_input/R2HDM_T1.ini । } \\
& \text { scan -n } 10
\end{aligned}
$$

to generate ten valid parameter points using the scan ranges in the example configuration file in the R2HDM and write them into an output file with the default name R2HDM.tsv (the same name as the executable with the extension . tsv added). All possible options are documented in the command line help which can be accessed e.g. through . / R2HDM -help (these include constraint severities and input parameter ranges). The configuration file simply specifies command line options. Since ScannerS performs a random scan, it is easily parallelizable by launching multiple jobs with the same input and different output files.

The output format used by ScannerS is a simple tabseparated tabular format where each line corresponds to one parameter point. The first column contains an index which identifies the point, while all following columns contain data. The first line contains a header that specifies the contents of each row. ${ }^{10}$ The pandas Python package for example can be used to easily read:

$$
\begin{array}{r}
d f=\text { pandas.read_table }(" R 2 H D M . t s v ", \\
\text { index_col=0) }
\end{array}
$$

and write:

df.to_csv("R2HDM_copy.tsv", sep=" \t" )

files in this format.

In check mode, the input parameters are read from a file. All of the input parameter points that fulfill the constraints are written to the output. This can e.g. be used to re-check a set of parameter points when the constraints have been updated. For example

. / R2HDM R2HDM_re.tsv check R2HDM. tsv

would re-check the parameter points generated in the previous example. Of course, unless the constraints changed between the two runs, R2HDM. tsv and R2HDM_re.tsv would contain the same parameter points. The check mode can also be used to apply the constraints to a manually specified set of parameter points, e.g. a parameter plane of interest. In models with multiple implemented sets of input parameters, check mode always uses the simplest possible input method - usually the input in terms of mixing angles - to minimize the numerical errors induced by repeated re-checks.

\subsection{Extending ScannerS}

ScannerS can easily be extended given some $\mathrm{C}++$ programming experience. New constraints can be added by extend-

\footnotetext{
10 The row names are documented with the function that stores the corresponding value to the output. This can either be one of the constraints in Scanners : : Constraints or a member function of the model class in ScannerS : : Models.
} 
ing the minimal example given in the documentation of the ScannerS: : Constraints: : Constraint class. The existing constraints illustrate a wide variety of possibilities. Some have almost trivial implementations that delegate all calculations to the model - such as the boundedness (ScannerS: : Constraints: :BFB) and unitarity (Scanners: : Constraints: :Unitarity) constraints - some perform calculations themselves - e.g. the constraint ScannerS: : Constraints: :STU that calculates and checks the oblique parameters - or delegate to external codes - like ScannerS: : Constraints: : Higgs, the constraint from Higgs observables, that calls HiggsBounds and HiggsSignals.

New models are also straightforward to implement. The main requirement is to implement the relations between the chosen set of input parameters and the remaining model parameters - e.g. the expressions for the $\lambda_{i}$ in terms of masses and mixing angles - in the constructor of a ParameterPoint member class. The basic technical requirements on a model class are listed in the documentation of the Scanners: : Models namespace.

Constraints are enabled for a model class by implementing their required functions and attributes. In most cases these are static member functions of the model class operating on a ParameterPoint object, the detailed requirements can be found in the documentation of each constraint in the Scanners: : Constraints namespace.

\section{Summary}

We have presented the $\mathrm{C}++$ code ScannerS that performs parameter scans in many BSM models with extended Higgs sectors. The resulting samples of parameter points can be used e.g. for phenomenological studies, as benchmark scenarios for experimental searches, or as numerical examples for precision calculations.

ScannerS implements many different sources of constraints on the models. These include theoretical constraints, constraints from precision measurements in the EW and flavour sector, constraints from LHC Higgs data, DM constraints, and EDM constraints as well as the requirement of a strong-first-order EW phase transition, if applicable. These constraints can be applied in parameter scans of many different implemented BSM models - different singlet extensions of the SM, both the CP-conserving and CP-violating 2HDM, and many different phases and variants of the N2HDM.

ScannerS aims to be easy to install - with automatic dependency management for the physics codes it uses - and easy to use - using a straightforward command line interface and simple tabular data files. We have used ScannerS to generate tens of millions of valid parameter points across the implemented models and the code proved to be reliable and efficient.
Implementing new constraints or models in ScannerS is straightforward and encouraged. The online documentation specifies the technical requirements and most extensions can be easily implemented by modifying and extending existing model or constraint implementations. If you have implemented new models or constraints in ScannerS we encourage you to contact us with regards to merging them back into the main code.

Acknowledgements We thank Duarte Azevado, Philipp Basler, Thomas Biekötter, Isabell Engeln, and Jonas Müller for using and testing pre-release versions of ScannerS and for providing important feedback. MM is supported by the BMBF-Project $05 \mathrm{H} 18 \mathrm{VKCC} 1$. RS acknowledges FCT support, Contracts UIDB/00618/2020, UIDP/00618/ 2020, PTDC/FIS-PAR/31. JW acknowledges funding by the European Research Council (ERC) under the European Union's Horizon 2020 research and innovation program, grant agreement No 668679 .

Data Availability Statement This manuscript has no associated data or the data will not be deposited. [Authors' comment: The ScannerS source code is publicly available at https://gitlab.com/jonaswittbrodt/ ScannerS.]

Open Access This article is licensed under a Creative Commons Attribution 4.0 International License, which permits use, sharing, adaptation, distribution and reproduction in any medium or format, as long as you give appropriate credit to the original author(s) and the source, provide a link to the Creative Commons licence, and indicate if changes were made. The images or other third party material in this article are included in the article's Creative Commons licence, unless indicated otherwise in a credit line to the material. If material is not included in the article's Creative Commons licence and your intended use is not permitted by statutory regulation or exceeds the permitted use, you will need to obtain permission directly from the copyright holder. To view a copy of this licence, visit http://creativecomm ons.org/licenses/by/4.0/.

Funded by $\mathrm{SCOAP}^{3}$.

\section{A The anyHdecay library}

In order to apply constraints from Higgs searches and Higgs measurements, precise model predictions for Higgs branching ratios and total widths are needed. ${ }^{11}$ ScannerS uses the interface library anyHdecay to obtain these predictions from dedicated tools based on the code HDECAY [29-31] that exist for various models. As these codes are not designed to be used as a library, anyHdecay wraps their functionality into a common $\mathrm{C}++$ interface.

The library currently incorporates HDECAY for the SM and R2HDM, sHDECAY [68] for the different phases of the CxSM (and the real-singlet-extended SM (RxSM) that is not currently implemented in ScannerS), N2HDECAY [82,84] for the phases of the N2HDM, and C2HDM_HDECAY [76] for the C2HDM. The anyHdecay source code is avail-

\footnotetext{
11 In simple models - such as pure singlet extensions - this can be circumvented by using the HiggsBounds effective coupling input [26].
} 
able at https://gitlab.com/jonaswittbrodt/anyhdecay and only requires working Fortran and $\mathrm{C}++-17$ compilers as well as CMake. The underlying codes are downloaded through CMake and automatically restructured to allow linking them into one library. The API documentation for anyHdecay is available at https://jonaswittbrodt.gitlab.io/anyhdecay/.

\section{B Perturbative unitarity bounds}

The tree-level $2 \rightarrow 2$ scattering matrix $\mathcal{M}_{2 \rightarrow 2}$ in the high energy limit is easy to derive directly from the scalar potential. An element of $\mathcal{M}_{2 \rightarrow 2}$ corresponding to a set of two particle states $|A B\rangle$ and $|C D\rangle$ is given by

$\langle A B|\mathcal{M}| C D\rangle=\frac{1}{\sqrt{\left(1+\delta_{A B}\right)\left(1+\delta_{C D}\right)}} \frac{\partial^{4} V}{\partial A \partial B \partial C \partial D}$,

where the $\delta$ functions lead to the correct symmetry factors for the two-particle states. The perturbative unitarity constraint bounds the $n$ eigenvalues $\mathcal{M}_{2 \rightarrow 2}^{i}(i \in\{1, \ldots, n\})$ of $\mathcal{M}_{2 \rightarrow 2}$ as

$\left|\mathcal{M}_{2 \rightarrow 2}^{i}\right|<8 \pi$.

However, since basis transformations are unitary transformations, the $\mathcal{M}_{2 \rightarrow 2}^{i}$ are independent of the basis [8], and the most convenient one - usually the basis of gauge eigenstates - can be used for the calculation.

The eigenvalues of $\mathcal{M}_{2 \rightarrow 2}$ can be calculated numerically in a straightforward way. However, in most cases the diagonalization can - at least partially - be carried out analytically. This both leads to additional insight into the allowed ranges for the parameters of the scalar potential and allows a much faster evaluation of perturbative unitarity constraints. A small Mathematica package tools/PerturbativeUnitarity.m - that can be used to perform this calculation is included in ScannerS. We will illustrate its usage by deriving the CxSM unitarity constraints of Eq. (11).

The package is loaded as usual (with the path adjusted as needed)

$<<$ tools / Perturbativeunitarity.m

It only needs the quartic part of the scalar potential - in terms of eigenstates of electric charge - and a list of fields as input. For the CxSM we define the quartic part of the scalar potential, Eq. (10), as

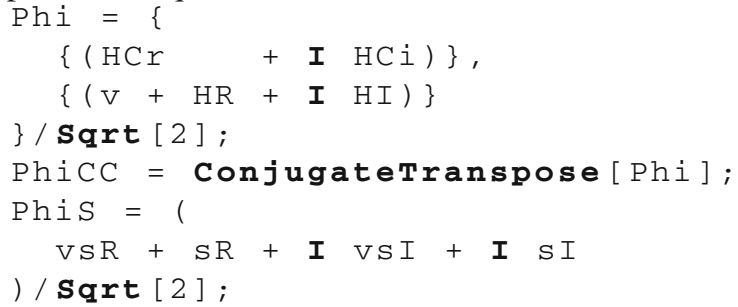

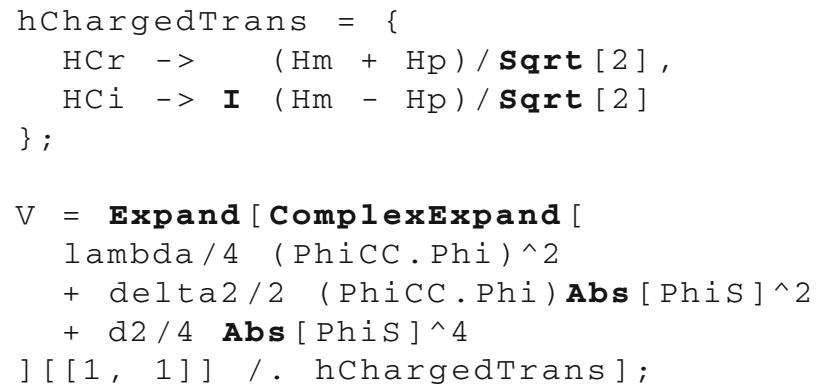

where the hChargedTrans rules are used to transform from the real field components into the complex electrically charged fields. The fields are defined as

fields $=\{\{\mathrm{Hp}, \mathrm{Hm}\}, \mathrm{HR}, \mathrm{HI}, \mathrm{sR}, \mathrm{SI}\}$;

where any charged fields should be given in a two-component sub-list with the positive eigenstate first and the negative eigenstate second. This information is used to avoid generating redundant charge conjugate states. With this input the package generates the scatter matrix $\mathcal{M}_{2 \rightarrow 2}$ through

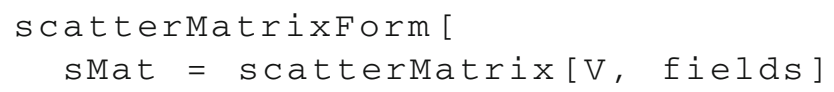

The scatterMatrixForm creates a formatted output of the - in case of the CXSM $16 \times 16$ - scattering matrix, where the corresponding two-particle states are indicated at each row and column. This full scatter matrix is decomposed into sub-blocks using

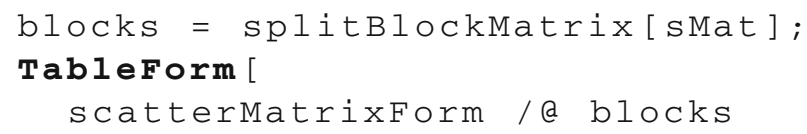

The second line prints all of the sub-matrices. In the CxSM this will show a $1 \times 1$ matrix of the doubly-charged states $\left|H^{ \pm} H^{ \pm}\right\rangle$, four $1 \times 1$ matrices for the singly-charged states, and another six $1 \times 1$ matrices and one $5 \times 5$ matrix for the neutral states. The eigenvalues $\mathcal{M}_{2 \rightarrow 2}^{i}$ are then obtained using

uniqueEv [ blocks ]

which gives the exact results of Eq. (11). The maximum and minimum values for the quartic potential parameters that are possible without violating unitarity may also be of interest. These can be obtained using

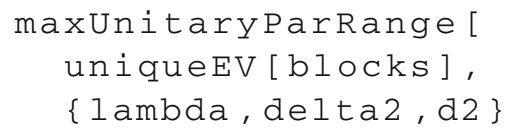

where the second argument lists the quartic parameters that enter the unitarity constraints. For the CXSM this returns that perturbative unitarity can only be fulfilled if 
$|\lambda| \lesssim 16.8, \quad\left|\delta_{2}\right| \lesssim 35.6, \quad\left|d_{2}\right| \lesssim 25.2$.

This information is particularly relevant to obtain reasonable scan ranges for models where some of the quartic parameters are input parameters - such as the dark phases of the N2HDM. The function works by numerically minimizing and maximizing each parameter given the perturbative unitarity constraints on all parameters. Optionally, additional constraints that should be included can be given as a third argument. For example, by including the CxSM boundedness conditions [1] as

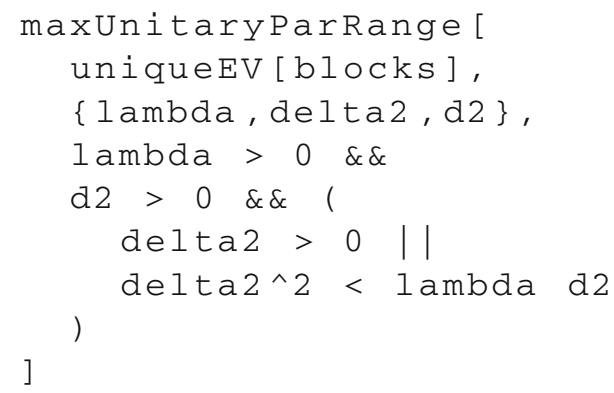

we obtain the smaller maximally allowed parameter ranges

$0 \leq \lambda \lesssim 16.8, \quad-13.1 \lesssim \delta_{2} \lesssim 35.6, \quad 0 \leq d_{2} \lesssim 25.2$

For more complicated models such as the N2HDM it is not possible to obtain all eigenvalues analytically. In this case some of the $\mathcal{M}_{2 \rightarrow 2}^{i}$ in the output of the uni queEV command will be expressed as the roots of a polynomial. These roots can then be calculated numerically, which is still substantially simpler than diagonalizing the full scatter matrix numerically - in the N2HDM the difference is between finding the roots of a cubic polynomial and diagonalizing a $40 \times 40$ scatter matrix. This exploding complexity is the reason, why ScannerS-2 no longer uses the generic numerical method for perturbative unitarity constraints [1] and instead includes this Mathematica package for easily obtaining (semi-)analytic conditions.

\section{References}

1. R. Coimbra, M.O.P. Sampaio, R. Santos, ScannerS: constraining the phase diagram of a complex scalar singlet at the LHC. Eur. Phys. J. C 73, 2428 (2013). https://doi.org/10.1140/epjc/ s10052-013-2428-4 arXiv:1301.2599

2. P. Bechtle, K. Desch, P. Wienemann, Fittino, a program for determining MSSM parameters from collider observables using an iterative method. Comput. Phys. Commun. 174, 47 (2006). https://doi. org/10.1016/j.cpc.2005.09.002 arXiv:hep-ph/0412012

3. A.B. Arbuzov et al., ZFITTER: a semi-analytical program for fermion pair production in $\mathrm{e}+\mathrm{e}-$ annihilation, from version 6.21 to version 6.42. Comput. Phys. Commun. 174, 728 (2006). https:// doi.org/10.1016/j.cpc.2005.12.009 arXiv:hep-ph/0507146

4. H. Flacher et al., Revisiting the global electroweak fit of thestandard model and beyond with Gfitter. Eur. Phys. J. C 60, 543
(2009). https://doi.org/10.1140/epjc/s10052-009-0966-6. https:// doi.org/10.1140/epjc/s10052-011-1718-y. arXiv:0811.0009

5. GAMBIT, GAMBIT: the global and modular beyond-thestandard-model in-ference tool. Eur. Phys. J. C 77, 784 (2017). https://doi.org/10.1140/epjc/s10052-017-5513-2. https://doi.org/ 10.1140/epjc/s10052-017-5321-8. arXiv:1705.07908

6. J. De Blas et al., HEPfit: a code for the combination of indirect and direct constraints on high energy physics models (2019). arXiv: 1910.14012

7. B.W. Lee, C. Quigg, H.B. Thacker, Weak interactions at very high-energies: the role of the Higgs Boson mass. Phys. Rev. D 16, 1519 (1977), FERMILAB-PUB-77-30-THY. https://doi.org/ 10.1103/PhysRevD.16.1519

8. S. Kanemura, T. Kubota, E. Takasugi, Lee-Quigg-Thacker bounds for Higgs boson masses in a two doublet model. Phys. Lett. B 313, 155 (1993). https://doi.org/10.1016/0370-2693(93)91205-2 arXiv:hep-ph/9303263

9. W.J. Marciano, G. Valencia, S. Willenbrock, Renormalization Group Im-proved unitarity bounds on the Higgs Boson and top quark masses. Phys. Rev. D 40, 1725 (1989), BNL-42766. https:// doi.org/10.1103/PhysRevD.40.1725

10. M.D. Goodsell, F. Staub, Unitarity constraints on general scalar couplings with SARAH. Eur. Phys. J. C 78, 649 (2018). https:// doi.org/10.1140/epjc/s10052-018-6127-z arXiv:1805.07306

11. M.D. Goodsell, F. Staub, Improved unitarity constraints in TwoHiggs-Doublet-Models. Phys. Lett. B 788, 206 (2019). https://doi. org/10.1016/j.physletb.2018.11.030 arXiv:1805.07310

12. M.E. Krauss, F. Staub, Unitarity constraints in triplet extensions beyond the large s limit. Phys. Rev. D 98, 015041 (2018). https:// doi.org/10.1103/PhysRevD.98.015041 arXiv:1805.07309

13. I.P. Ivanov, M. Köpke, M. Mühlleitner, Algorithmic boundednessfrom-below conditions for generic scalar potentials. Eur. Phys. J. C 78, 413 (2018). https://doi.org/10.1140/epjc/s10052-018-5893-y arXiv: 1802.07976

14. W.G. Hollik, G. Weiglein, J. Wittbrodt, Impact of vacuum stability constraints on the phenomenology of supersymmetric models. JHEP 03, 109 (2019). https://doi.org/10.1007/JHEP03(2019)109 arXiv: 1812.04644

15. P.M. Ferreira et al., Vacuum instabilities in the N2HDM. J. High Energy Phys. (2019). https://doi.org/10.1007/JHEP09(2019)006. arXiv:1905.10234

16. J. Wittbrodt, EVADE: Efficient constraints from Vacuum DEcay. http://gitlab.com/jonaswittbrodt/evade

17. M.E. Peskin, T. Takeuchi, Estimation of oblique electroweak corrections. Phys. Rev. D 46, 381 (1992), SLAC-PUB-5618. https:// doi.org/10.1103/PhysRevD.46.381

18. J. Haller et al., Update of the global electroweak fit and constraints on two-Higgs-doublet models. Eur. Phys. J. C 78, 675 (2018). https://doi.org/10.1140/epjc/s10052-018-6131-3 arXiv: 1803.01853

19. W. Grimus et al., A precision constraint on multi-Higgs-doublet models. J. Phys. G35, 075001 (2008). https://doi.org/10.1088/ 0954-3899/35/7/075001 arXiv:0711.4022

20. W. Grimus et al., The oblique parameters in multi-Higgs-doublet models. Nucl. Phys. B 801, 81 (2008). https://doi.org/10.1016/j. nuclphysb.2008.04.019 arXiv:0802.4353

21. A.J. Buras et al., Higgs-mediated FCNCs: natural flavour conservation vs minimal flavour violation. JHEP 10, 009 (2010). https:// doi.org/10.1007/JHEP10(2010)009 arXiv:1005.5310

22. P. Bechtle et al., HiggsBounds: confronting arbitrary Higgs sectors with exclusion bounds from LEP and the Tevatron. Comput. Phys. Commun. 181, 138 (2010). https://doi.org/10.1016/j.cpc.2009.09. 003 arXiv:0811.4169

23. P. Bechtle et al., HiggsBounds 2.0.0: confronting neutral and charged higgs sector predictions with exclusion bounds from LEP 
and the Tevatron. Comput. Phys. Commun. 182, 2605 (2011). https://doi.org/10.1016/j.cpc.2011.07.015 arXiv:1102.1898

24. P. Bechtle et al., HiggsBounds-4: improved tests of extended Higgs sectors against exclusion bounds from LEP, the Tevatron and the LHC. Eur. Phys. J. C 74, 2693 (2014). https://doi.org/10.1140/epjc/ s10052-013-2693-2 arXiv:1311.0055

25. P. Bechtle et al., Applying exclusion likelihoods from LHC searches to extended Higgs sectors. Eur. Phys. J. C 75, 421 (2015). https://doi.org/10.1140/epjc/s10052-015-3650-z arXiv:1507.06706

26. P. Bechtle et al., HiggsBounds-5: testing Higgs sectors in the LHC13 TeV Era (2020). arXiv:2006.06007

27. P. Bechtle et al., HiggsSignals: confronting arbitrary Higgs sectors with measurements at the Tevatron and the LHC. Eur. Phys. J. C 74, 2711 (2014). https://doi.org/10.1140/epjc/s10052-013-2711-4 arXiv: 1305.1933

28. P. Bechtle et al., HiggsSignals-2: probing new physics with precision Higgs measurements in the LHC $13 \mathrm{TeV}$ era. Eur. Phys. J. C 81, 145 (2021). https://doi.org/10.1140/epjc/s10052-021-08942-y arXiv:2012.09197

29. A. Djouadi, J. Kalinowski, M. Spira, HDECAY: a program for Higgs boson decays in the standard model and its supersymmetric extension. Comput. Phys. Commun. 108, 56 (1998). https://doi. org/10.1016/S0010-4655(97)00123-9 arXiv:hep-ph/9704448

30. R. Harlander et al., Interim recommendations for the evaluation of Higgs production cross sections and branching ratios at the LHC in the Two-Higgs-Doublet Model (2013). arXiv:1312.5571

31. A. Djouadi et al., HDECAY: twenty++ years after. Comput. Phys. Commun. 238, 214 (2019). https://doi.org/10.1016/j.cpc.2018.12. 010 arXiv:1801.09506

32. R.V. Harlander, S. Liebler, H. Mantler, SusHi: a program for the calculation of Higgs production in gluon fusion and bottom-quark annihilation in the Standard Model and the MSSM. Comput. Phys. Commun. 184, 1605 (2013). https://doi.org/10.1016/j.cpc.2013. 02.006 arXiv: 1212.3249

33. R.V. Harlander, S. Liebler, H. Mantler, SusHi Bento: beyond NNLO and the heavy-top limit. Comput. Phys. Commun. 212, 239 (2017). https://doi.org/10.1016/j.cpc.2016.10.015 arXiv: 1605.03190

34. O. Brein, R.V.Harlander, T.J.E.Zirke, vh@nnlo-Higgs Strahlung at hadron colliders. Comput. Phys. Commun. 184, 998 (2013). https:// doi.org/10.1016/j.cpc.2012.11.002 arXiv:1210.5347

35. R.V.Harlander et al.,vh@ nnlo-v2: new physics in Higgs Strahlung. JHEP 05, 089 (2018). https://doi.org/10.1007/JHEP05(2018)089 arXiv: 1802.04817

36. E.L. Berger et al., Associated production of a top quark and a charged Higgs boson. Phys. Rev. D 71, 115012 (2005). https://doi. org/10.1103/PhysRevD.71.115012 arXiv:hep-ph/0312286

37. S. Dittmaier et al., Charged-Higgs-boson production at the LHC: NLO supersymmetric QCD corrections. Phys. Rev. D 83, 055005 (2011). https://doi.org/10.1103/PhysRevD.83.055005 arXiv:0906.2648

38. M. Flechl et al., Improved cross-section predictions for heavy charged Higgs boson production at the LHC. Phys. Rev. D 91, 075015 (2015). https://doi.org/10.1103/PhysRevD.91.075015 arXiv: 1409.5615

39. C. Degrande et al., Heavy charged Higgs boson production at the LHC. JHEP 10, 145 (2015). https://doi.org/10.1007/ JHEP10(2015)145 arXiv:1507.02549

40. LHC Higgs Cross Section Working Group, Handbook of LHC Higgs Cross Sections: 4. Deciphering the Nature of the Higgs Sector (2016). https://doi.org/10.23731/CYRM-2017-002. arXiv: 1610.07922

41. C. Degrande et al., Accurate predictions for charged Higgs production: closing the $m_{H} \pm \sim m_{t}$ window. Phys. Lett. B 772, 87 (2017). https://doi.org/10.1016/j.physletb.2017.06.037 arXiv:1607.05291
42. ACME, Improved limit on the electric dipole moment of the electron. Nature 562, 355 (2018). https://doi.org/10.1038/ s41586-018-0599-8

43. G. Belanger et al., MicrOMEGAs 2.0: a program to calculate the relic density of dark matter in a generic model. Comput. Phys. Commun. 176, 367 (2007). https://doi.org/10.1016/j.cpc.2006.11. 008 arXiv:hep-ph/0607059

44. G. Belanger et al., Dark matter direct detection rate in a generic model with micrOMEGAs 2.2. Comput. Phys. Commun. 180, 747 (2009). https://doi.org/10.1016/j.cpc.2008.11.019 arXiv:0803.2360

45. G. Belanger et al., Indirect search for dark matter with micrOMEGAs2 4. Comput. Phys. Commun. 182, 842 (2011). https://doi.org/10.1016/j.cpc.2010.11.033 arXiv:1004.1092

46. G. Belanger et al., micrOMEGAs 3: a program for calculating dark matter observables. Comput. Phys. Commun. 185, 960 (2014). https://doi.org/10.1016/j.cpc.2013.10.016 arXiv:1305.0237

47. G. Bélanger et al., micrOMEGAs4.1: two dark matter candidates. Comput. Phys. Commun. 192, 322 (2015). https://doi.org/10.1016/ j.cpc.2015.03.003 arXiv:1407.6129

48. D. Barducci et al., Collider limits on new physics within micrOMEGAs 4.3. Comput. Phys. Commun. 222, 327 (2018). https://doi.org/10.1016/j.cpc.2017.08.028 arXiv:1606.03834

49. G. Bélanger et al., micrOMEGAs5.0: freeze-in. Comput. Phys. Commun. 231, 173 (2018). https://doi.org/10.1016/j.cpc.2018.04. 027 arXiv: 1801.03509

50. Planck, Planck 2018 results. VI. Cosmological parameters (2018). arXiv: 1807.06209

51. XENON, Dark matter search results from a one ton-year exposure of XENON1T. Phys. Rev. Lett. 121, 111302 (2018). https://doi. org/10.1103/PhysRevLett.121.111302. arXiv:1805.12562

52. V.A. Kuzmin, V.A. Rubakov, M.E. Shaposhnikov, On the anomalous electroweak baryon number nonconservation in the early universe. Phys. Lett. B 155, 36, IC/85/8 (1985). https://doi.org/10. 1016/0370-2693(85)91028-7

53. M.E. Shaposhnikov, Possible appearance of the baryon asymmetry of the universe in an electroweak theory. JETP Lett. 44, 465 (1986)

54. M. Trodden, Electroweak baryogenesis. Rev. Mod. Phys. 71, 1463 (1999). https://doi.org/10.1103/RevModPhys.71.1463 arXiv:hep-ph/9803479

55. D.E. Morrissey, M.J. Ramsey-Musolf, Electroweak baryogenesis. New J. Phys. 14, 125003 (2012). https://doi.org/10.1088/ 1367-2630/14/12/125003 arXiv:1206.2942

56. A. Kosowsky, M.S. Turner, R. Watkins, Gravitational waves from first order cosmological phase transitions. Phys. Rev. Lett. 69, 2026 (1992) FERMILAB-PUB-91-333-A-REV. https://doi.org/ 10.1103/PhysRevLett.69.2026

57. A. Kosowsky, M.S. Turner, R. Watkins, Gravitational radiation from colliding vacuum bubbles. Phys. Rev. D 45, 4514 (1992), FERMILAB-PUB-91-323-A. https://doi.org/10.1103/PhysRevD. 45.4514

58. A. Kosowsky, M.S. Turner, Gravitational radiation from colliding vacuum bubbles: envelope approximation to many bubble collisions. Phys. Rev. D 47, 4372 (1993). https://doi.org/10.1103/ PhysRevD.47.4372 arXiv:astro-ph/9211004

59. M. Kamionkowski, A. Kosowsky, M.S. Turner, Gravitational radiation from first order phase transitions. Phys. Rev. D 49, 2837 (1994). https://doi.org/10.1103/PhysRevD.49.2837 arXiv:astro-ph/9310044

60. R. Apreda et al., Gravitational waves from electroweak phase transitions. Nucl. Phys. B 631, 342 (2002). https://doi.org/10.1016/ S0550-3213(02)00264-X arXiv:gr-qc/0107033

61. A. Kosowsky, A. Mack, T. Kahniashvili, Gravitational radiation from cosmological turbulence. Phys. Rev. D 66, 024030 (2002). https://doi.org/10.1103/PhysRevD.66.024030 arXiv:astro-ph/0111483 
62. A.D. Dolgov, D. Grasso, A. Nicolis, Relic backgrounds of gravitational waves from cosmic turbulence. Phys. Rev. D 66, 103505 (2002). https://doi.org/10.1103/PhysRevD.66.103505 arXiv:astro-ph/0206461

63. C. Grojean, G. Servant, Gravitational waves from phase transitions at the electroweak scale and beyond. Phys. Rev. D 75, 043507 (2007). https://doi.org/10.1103/PhysRevD.75.043507. arXiv:hep-ph/0607107

64. C. Caprini et al., Science with the space-based interferometer eLISA. II: Gravitational waves from cosmological phase transitions. JCAP 1604, 001 (2016). https://doi.org/10.1088/1475-7516/ 2016/04/001. arXiv:1512.06239

65. P. Basler, M. Mühlleitner, BSMPT (Beyond the Standard Model Phase Transitions): a tool for the electroweak phase transition in extended Higgs sectors. Comput. Phys. Commun. 237, 62 (2019). https://doi.org/10.1016/j.cpc.2018.11.006 arXiv:1803.02846

66. P. Basler, M. Mühlleitner, J. Müller, BSMPT v2 a tool for the electroweak phase transition and the baryon asymmetry of the universe in extended Higgs sectors (2020). arXiv:2007.01725

67. V. Barger et al., Complex singlet extension of the standard model. Phys. Rev. D 79, 015018 (2009). https://doi.org/10.1103/ PhysRevD.79.015018 arXiv:0811.0393

68. R. Costa et al., Singlet extensions of the standard model at LHCRun 2: bench-marks and comparison with the NMSSM. JHEP 06, 034 (2016). https://doi.org/10.1007/JHEP06(2016)034 arXiv: 1512.05355

69. T. Robens, T. Stefaniak, J. Wittbrodt, Two-real-scalar-singlet extension of the SM: LHC phenomenology and benchmark scenarios (2019). arXiv: 1908.08554

70. T.D. Lee, A theory of spontaneous T violation. Phys. Rev. D 8, 1226 (1973). https://doi.org/10.1103/PhysRevD.8.1226

71. G.C. Branco et al., Theory and phenomenology of two-Higgsdoublet models. Phys. Rep. 516, 1 (2012). https://doi.org/10.1016/ j.physrep.2012.02.002 arXiv:1106.0034

72. G.C. Branco, M.N. Rebelo, The Higgs mass in a model with two scalar doublets and spontaneous $\mathrm{CP}$ violation. Phys. Lett. B 160, 117 (1985), IFM-7/85. https://doi.org/10.1016/ 0370-2693(85)91476-5

73. I.F. Ginzburg, M. Krawczyk, P. Osland, Two Higgs doublet models with CP violation. In: Linear colliders, Seogwipo, Korea, August 26-30, pp. 703-706 (2002). arXiv:hep-ph/0211371

74. W. Khater, P. Osland, CP violation in top quark production at the LHC and two Higgs doublet models. Nucl. Phys. B 661, 209 (2003). https://doi.org/10.1016/S0550-3213(03)00300-6 arXiv:hep-ph/0302004

75. A. Barroso et al., Metastability bounds on the two Higgs doublet model. JHEP 06, 045 (2013). https://doi.org/10.1007/ JHEP06(2013)045 arXiv:1303.5098

76. D. Fontes et al., The C2HDM revisited. JHEP 02, 073 (2018). https://doi.org/10.1007/JHEP02(2018)073 arXiv:1711.09419
77. I.P. Ivanov, J.P. Silva, Tree-level metastability bounds for the most general two Higgs doublet model. Phys. Rev. D 92, 055017 (2015). https://doi.org/10.1103/PhysRevD.92.055017 arXiv:1507.05100

78. S. Liebler, S. Patel, G. Weiglein, Phenomenology of on-shell Higgs production in the MSSM with complex parameters. Eur. Phys. J. C 77, 305 (2017). https://doi.org/10.1140/epjc/s10052-017-4849-y arXiv: 1611.09308

79. T. Abe et al., Gauge invariant Barr-Zee type contributions to fermionic EDMs in the two-Higgs doublet models. JHEP 01, 106 (2014). https://doi.org/10.1007/JHEP01(2014)106,10.1007/ JHEP04(2016)161 arXiv:1311.4704

80. C.-Y. Chen, M. Freid, M. Sher, Next-to-minimal two Higgs doublet model. Phys. Rev. D 89, 075009 (2014). https://doi.org/10.1103/ PhysRevD.89.075009 arXiv:1312.3949

81. A. Drozd et al., Extending two-Higgs-doublet models by a singlet scalar field-the Case for Dark Matter. JHEP 11, 105 (2014). https:// doi.org/10.1007/JHEP11(2014)105 arXiv:1408.2106

82. M. Mühlleitner et al., The N2HDM under theoretical and experimental scrutiny. JHEP 03, 094 (2017). https://doi.org/10.1007/ JHEP03(2017)094 arXiv:1612.01309

83. K.G. Klimenko, On necessary and sufficient conditions for some Higgs potentials to be bounded from below. Theor. Math. Phys. 62, 58 (1985), IFVE-84-43. https://doi.org/10.1007/BF01034825

84. I. Engeln, M. Mühlleitner, J. Wittbrodt, N2HDECAY: Higgs boson decays in the different phases of the N2HDM. Comput. Phys. Commun. 234, 256 (2018). https://doi.org/10.1016/j.cpc.2018.07.020 arXiv:1805.00966

85. P. Basler, M. Mühlleitner, J. Müller, Electroweak phase transition in nonminimal Higgs sectors. JHEP 05, 016 (2020). https://doi. org/10.1007/JHEP05(2020)016 arXiv:1912.10477

86. I. Engeln, Phenomenological comparison of the dark phases of the next-to-two-Higgs-doublet model, MA thesis, KIT, Karlsruhe (2018). https://www.itp.kit.edu/_media/publications/ masterthesis_isabellengeln.pdf

87. I. Engeln et al., The dark phases of the N2HDM (2020). arXiv:2004.05382

88. N.G. Deshpande, E. Ma, Pattern of symmetry breaking with two Higgs doublets. Phys. Rev. D 18, 2574 (1978), OITS-81. https:// doi.org/10.1103/PhysRevD.18.2574

89. D. Azevedo et al., CP in the dark. JHEP 11, 091 (2018). https:// doi.org/10.1007/JHEP11(2018)091 arXiv:1807.10322

90. M. Galassi et al., GNU Scientific Library Reference Manual, 3rd ed. (Network Theory Limited, Bristol, 2009)

91. G. Guennebaud, B. Jacob et al., Eigen v3 (2010). https://eigen. tuxfamily.org 